enhances development and post-transfer survival of in vitro-produced bovine embryos. Holstein COC shipped overnight in a portable incubator in oocyte maturation medium were fertilized with X-chromosome selected sperm from Holstein bulls. X-selected sperm were used because females were preferred. Morulae and blastocysts were collected at Day 7 after insemination and transferred at Day 7 after ovulation to lactating dairy cows subjected to a modified OvSynch protocol. In Experiment 1, conducted from June 29 to August 31, embryos were cultured in KSOM-BE2 alone, KSOM-BE2 with $100 \mathrm{ng} \mathrm{mL}^{-1}$ of $\mathrm{Arg}^{3}$-IGF-1 or KSOM-BE2 with $10 \mathrm{ng} \mathrm{mL}^{-1}$ of recombinant BoGM-CSF. Treatments were added at Day 1 after insemination. As compared to control embryos $(17 \pm 2 \%)$, the percentage of cleaved embryos that became transferable morulae or blastocysts at Day 7 was increased $(P<0.05)$ by GM-CSF $(25 \pm 2 \%)$ but not by Arg $^{3}$-IGF-1 $(18 \pm 2 \%)$. There was no significant effect of treatment on pregnancy rate at Day 30 to $35[34 \%(n=52), 35 \%(n=51)$, and $43 \%(n=55)$ for control, GM-CSF, and IGF-1, respectively] or calving rate $(27,35$, and $40 \%)$ although values were numerically greater for cows receiving IGF-1 treated embryos. In experiment 2, conducted from September 7 to February 1 , embryos were cultured in KSOM-BE2 alone, KSOM-BE2 with $100 \mathrm{ng} \mathrm{mL}^{-1} \mathrm{Arg}^{3}$-IGF-1 added at Day 1 after insemination, or KSOM-BE2 with $10 \mathrm{ng} \mathrm{mL}^{-1}$ recombinant BoGM-CSF added at Day 5 after insemination. GM-CSF, but not IGF-1, increased the percentage of oocytes $(P<0.03)$ and the percentage of cleaved embryos $(P=0.05)$ that became transferable morulae or blastocysts at Day 7 . The percentage of cleaved embryos

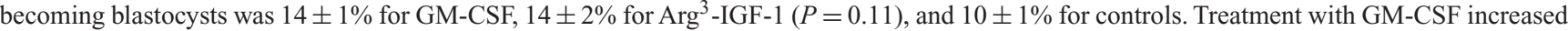
$(P=0.056)$ the percentage of cows pregnant at Day 30 to $35[34 \%(n=79), 43 \%(n=107)$, and $27 \%(n=44)$ for control, GM-CSF, and IGF-1, respectively]. Data on calving rate are currently being collected; to date, $86 \%$ of calves were female. Results indicate that embryo competence for post-transfer survival can be enhanced by treatment with GM-CSF at Day 5 after fertilization.

Research supported by USDA Grant 2006-55203-17390, BARD Grant US-3986-07 and the Southeast Milk Dairy Checkoff Program. B.L. was supported by a CAPES (Brazil)/Fulbright Fellowship.

\title{
143 SUPEROVULATORY RESPONSE AND EMBRYO PRODUCTION INFLUENCED BY THE ADDITION OF LH AND EFFECT OF THE REPEATABILITY IN SANTA INÊS SHEEP
}

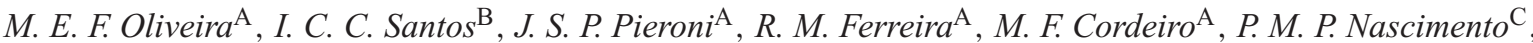 \\ S. F. Souza ${ }^{\mathrm{A}}$, J. F. Fonseca ${ }^{\mathrm{C}}$, and W. R. R. Vicente ${ }^{\mathrm{A}}$ \\ A UNESP, Jaboticabal, Sao Paulo, Brazil; \\ ${ }^{B}$ Tecnopec ltda, Sao Paulo, Sao Paulo, Brazil; \\ ${ }^{\mathrm{C}}$ Embrapa Caprinos, Sobral, Ceara, Brazil
}

The aim this study was to evaluate the effect of the addition of LH in superovulatory response and embryo production in Santa Inês sheep. Ten donors with $60.3 \pm 10.7 \mathrm{~kg}$ and BCS of $3.9 \pm 0.3$ were superovulated in a cross-over design, with a 60-day interval. Estrus was synchronized with a progesterone-releasing intravaginal device (CIDR ${ }^{\mathrm{TM}}$; Pfizer Animal Health, Brazil) inserted on Day 0 and replaced by a new one on Day 7 , that was maintained to Day 14. Two doses of $37.5 \mathrm{~g}$ of D-cloprostenol (Prolise ${ }^{\mathrm{TM}}$, Arsa, Buenos Aires, Argentina) were administered, on Days 7 and 14. Donors also receive $256 \mathrm{mg}$ of pFSH (Folltropin ${ }^{\mathrm{TM}}$, Bioniche, Belleville, ON, Canada) in 8 decreasing doses, starting on Day 12. On Day 14, all females received 200 IU of eCG (Novormon TM, Syntex, Argentina). On Day 15, the animals were homogeneously allocated in 1 of 2 groups: Control (GC, $n=10)$ and treated (G-LH, $n=10)$. Ewes in GC did not receive exogenous LH, whereas ewes in G-LH were treated with $7.5 \mathrm{mg}$ of LH (Lutropin $^{\mathrm{TM}}$, Bioniche), on Day 15. All females were inseminated by laparoscopy, with frozen-thawed semen, 42 and $48 \mathrm{~h}$ after CIDR removal. On Day 21, the embryos were surgically collected. The superovulatory response was classified in scores: (0) 4 or fewer CL; (1) between 5 and 10 CL, and (2) 11 or more CL. Means were compared using Kruskal-Wallis test and percentages using chi-square $(P<0.05)$. Most of donors $(70 \%, 7 / 10)$ from G-LH presented a superovulatory response classified as score 2 , and the remaining $(30 \%, 3 / 10)$ as score 1 , whereas, half of the controls were classified as score 2 and half as score 1. Ovulation rate tended to be greater in G-LH $(135 / 158,85.4 \% v .105 / 135,77.7 \%, P=0.08)$. The number of $\mathrm{CL}($ mean $\pm \mathrm{SD}$ ) was $10.5 \pm 3.8$ in GC and $13.5 \pm 4.84$ in G-LH, but was not statistically different. The number of anovulatory follicles (AF) did not differ between groups (GC: $3.0 \pm 3.2$; G-LH: $2.3 \pm 1.6$ ), but the proportion of AF tended to decrease in G-LH $(30 / 135,22.2 \% v .23 / 158,14.5 \%$, $P=0.08)$. Considering embryo production, there was no difference between GC and G-LH $(P>0.05)$ related to number of recovered ova/embryos $(6.1 \pm 4.6 v .8 .4 \pm 5.2)$, viable embryos $(3.8 \pm 4.3 v .4 .2 \pm 5.2)$, unfertilized $(1.7 \pm 3.4 v .2 .0 \pm 2.9)$ and degenerated embryos $(0.7 \pm 0.7 v .2 .2 \pm 2.9)$ respectively. Data showed that the addition of LH tended to increase ovulation rate and to decrease the proportion of AF, but did not affect the number of viable embryos.

\section{PREGNANCY RETENTION OF BOVINE RECIPIENTS FOLLOWING TRANSFER OF EMBRYOS EXPOSED TO A PROSTAGLANDIN ${ }_{2 \alpha}$ RECEPTOR ANTAGONIST DURING COLLECTION}

\author{
D. A. Roper, F. N. Scenna, A. M. Saxton, J. L. Edwards, N. R. Rohrbach, and F. N. Schrick
}

Department of Animal Science, The University of Tennessee, Knoxville, TN

Increasing efficiency and success of MOET continues to be a goal of researchers and practitioners. Although numerous studies report success in establishing pregnancies, fewer evaluate term development and report number of live calves born. In a previous study, Scenna FN et al. (2008 Reprod. Fertil. Dev. 20, 154) exposed embryos to 3 different medium treatments while being collected from superovulated beef donors on Day 7. Medium treatments consisted of a commercially available medium plus $1 \mathrm{~mL}$ of DMSO (Control), commercial medium plus $100 \mathrm{~nm}$ of AL-8810 (AL100), or a commercial medium plus $1000 \mathrm{~nm}$ of AL-8810 (AL1000). Embryos were evaluated for grade and stage according to IETS guidelines. Embryos ( $n=1734$ at 6 locations across 13 replicates) were transferred (fresh or frozen in ethylene glycol) by 4 experienced technicians. Pregnancy rates were determined by ultrasonography 28 to 35 days after transfer and were increased in recipients receiving embryos collected in media containing 